\title{
Open Collaboration in an Age of Distrust
}

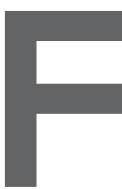

OR OVER 30 years, computing has been pursued in an environment of trust with computing research advances and publications shared openly within a truly integrated international community. At the heart is the explosive 20-year rise of open source software shared touchstones sufficient to build enterprise-scale software systems and giving rise to multibillion-dollar companies and entire new service sectors.

The bounty of open sharing is the rapid advance of computing technologies - the Internet, WWW, and a wide variety of Internet and cloud services. Equally important, open source sharing has been a boon for education, building an open international community that included developed countries in Europe and North America as well as developing countries such as Brazil, Russia, India, and China. All have contributed and benefitted tremendously in return.

The global backdrop for computing's open sharing was an environment of international trust and secular trend toward global integration of economy and society. We are manifestly in a new era of international relations-"An Age of Distrust" -where the trend toward increased trade and integration has stalled, if not reversed. And, a new superpower competition between the U.S. and China for global scientific, economic, and other forms of leadership is reshaping perspective and strategy. ${ }^{\mathrm{b}}$

It is time for the computing community to begin thinking and discussing

a S. Phipps. Open source software: 20 years and counting, (Feb. 3, 2018), opensource.com

b China v America: The end of engagement, how the world's two superpowers have become rivals. Economist, (Oct. 18, 2018); J. Perlez. U.S.China clash at Asian summit was over more than words. NY Times, (Nov. 19, 2018). what it means to engage in open collaboration in an Age of Distrust. Why must the computing community change?

While computing has supported military technology (design) and tactics (gunnery tables) from its earliest days, ${ }^{\mathrm{c}}$ they were not the direct tools of aggression. The evidence is undeniable that computing is now a dual-use technology with capability for direct aggression.

- Cybersecurity technologies are used extensively as instruments of aggression by governments and non-governmental organizations for industrial espionage, sabotage, and subversion of elections, ${ }^{\mathrm{d}}$ and even entire countries' infrastructure. Cybersecurity technology is used for asymmetric attacks on the wealthy and powerful-nations, companies, CEO's, but can also be turned on the poor, weak, and individuals.

- Artificial intelligence technologies have growing capabilities for surveillance, espionage, and more intimidating potential to create autonomous and robotic systems. So serious are these concerns that leading AI researchers have called for a ban on development of autonomous weapons, ${ }^{\mathrm{e}}$ and others have protested and prevented their company's participation in military applications. ${ }^{\mathrm{f}}$ Most countries believe AI is not only commercially important, but also strategic for intelligence and warfare cyberspace and the physical world.

c History of Computing Hardware; https://bit.ly/2IHzgP4.

d M.S. Schmidt and D.E. Sanger. 5 in China army face U.S. charges of cyberattack. NY Times, (May 19, 2014). A. Greenberg. How an entire nation became Russia's test lab for cyberwar. WIRED, (June 20, 2017); The untold story of NOTPETYA, the most devastating cyberattack in history. WIRED, (Aug. 22, 2018).

e Autonomous weapons: An open letter from AI \& robotics researchers; https://futureoflife. org/open-letter-autonomous-weapons/
Furthermore, computing's unique capability for instantaneous translation from commercial to military use-download, build, and incorporate-make traditional notions of control' ${ }^{g}$ irrelevant.

Companies face increasing assertion of national sovereignty and controlgovernment access to data, citizen data privacy rights, even information control. ${ }^{\mathrm{h}}$ Universities and research institutes face increasing questions about whom to collaborate with, to share information with, and to allow to work on projects. At issue is the ethical and moral implications of research. Export control regulations proliferate, "deemed export" is increasingly challenging, and new regulations controlling information sharing and research seem likely.

Within science, the physics community has faced these concerns for much of the $20^{\text {th }}$ century, and recently so has the biology community. Within computing, the cryptography community is no stranger to these concerns. We should seek to learn from them.

Let me be clear, I am not advocating banning, control, or classification of research topics. The computing community is too large and international for any single country or organization to limit the progress in computing technologies. However, such efforts will inevitably arise, so we, as computing professionals, must begin the difficult conversations of how to shape the development and use of technologies so that they can be a responsible and accountable force in society.

Let's begin the conversation! C

Andrew A. Chien, EDITOR-IN-CHIE F

f D. Wakabayashi and S. Shane. Google will not renew Pentagon contract that upset employees. NY Times, (June 1, 2018).

g UN Office for Disarmament Affairs. Treaty on the Non-Proliferation of Nuclear Weapons; https://bit.ly/2gxxd2j

$h$ E.C. Economy. The great firewall of China: Xi Jinping's Internet shutdown, The Guardian, (June 29, 2018) and European Union: General data protection regulation; https://gdpr-info.eu/ 